\title{
New Model for Solving Mixed Integral Equation of the First Kind with Generalized Potential Kernel
}

\author{
Shareefa Eisa Ali Alhazmi ${ }^{1}$ \\ ${ }^{1}$ Department of Mathematics Faculty of Science Umm Al-Qura University, Saudi Arabia \\ Correspondence: Department of Mathematics Faculty of Science Umm Al-Qura University, Saudi Arabia. \\ E-mail: sehazmi@uqu.edu.sa
}

Received: June 5, 2017 Accepted: June 23, 2017 Online Published: August 21, 2017

doi:10.5539/jmr.v9n5p18 URL: https://doi.org/10.5539/jmr.v9n5p18

\begin{abstract}
New technique model is used to solve the mixed integral equation (MIE) of the first kind, with a position kernel contains a generalized potential function multiplying by a continuous function and continuous kernel in time, in the space $L_{2}(\Omega) \times$ $C[0, T], 0 \leq T<1, \Omega$ is the domain of integration and $T$ is the time. The integral equation arises in the treatment of various semi-symmetric contact problems, in one, two, and three dimensions, with mixed boundary conditions in the mechanics of continuous media. The solution of the MIE when the kernel of position takes the potential function form, elliptic function form, Carleman function and logarithmic kernel are discussed and obtain in this work. Moreover, many special cases are derived.
\end{abstract}

Keywords: Mixed integral equation, Contact problems, Generalized potential kernel, Hypergeometric function. Bessel function

\section{Introduction}

The field of integral and integro-differential equations is a very important subject in applied mathematics, because mathematical formulation of many physical phenomena contains integral and integro-differential equations (Saeedi, L., Tari, A. \& Masuleh, S. H., 2013).Integral equations are very important branch of mathematics, which come in application in many physical problems. Now, the integral equations have received considerable interest of many applications in different mathematical areas of sciences, for example see (Saeedi, L., Tari, A. \& Masuleh, S. H., 2013; Geng, F. Z. \& Qian, S. P. 2015; Mohammadi, M. \& Mokhtari, R., 2011; Mohammadi, M. \& Mokhtari, R., 2013; Diego, T. \& Lima, P., 2008).Therefore, different analytic and numeric methods have been established to obtain the solutions of the integral equations. For analytical methods, we state degenerate kernel method, Cauchy method, Laplace transformation method, Fourier transformation method, potential theory method, and Krien's method. More information for the analytic methods can be found in Muskhelishvili (2002), Popov (1982), Tricomi (1985), Hochstad (1971) and Green (Dzhuraev, 1992).

When the analytic solution fails, it is often necessary to use of numerical techniques, which are appropriate combination of numerical integration and interpolation (Linz, P., 1985). The authors of (Ordokhani, Y. \& Dehestani, H., 2010) used Walsh functions operational matrix with Newton-Cotes nodes for solving Fredholm-Hammerstein integro-differential equations. In (Ordokhani, Y., 2013), a collocation method based on the Bessel polynomials was used for solution of the nonlinear Fredholm-Volterra Hammerstein integro-differential equations, under mixed conditions. In (Turkyilmazoglu, M., 2014) high-order linear Fredholm integro-differential equations were solved by means of an elegant and accurate effective technique. In (Gouyandeh, Z., Allahviranloo, T. \& Armand, A., 2016), the approximate solution for the nonlinear Volterra-Fredholm-Hammerste in integral equations was obtained by using the Tau-collocation method. In (Parand, K. \& Rad, J. A., 2012), a numerical technique based on the spectral method was presented to solve the nonlinear VolterraFredholm-Hammerstein integral equations

The interested reader should consult the fine exposition by Atkinson ( 1976), Delves and Mohamed (Delves, L. M. \& Mohamed, J. L., 1985), and Golberg (Golberg, M. A., 1990) for some different numerical methods.

In (Abdou, M. A., 2002); Abdou, M. A., 2000)),MIEs of the second kind with different singular kernels, in position are considered, under certain conditions. Moreover, using different methods the solution, in each case, is obtained.

Consider the following MIE:

$$
\begin{aligned}
& \iint_{\Omega} L(\xi, \eta)\left[(x-\xi)^{2}+(y-\eta)^{2}\right]^{-h} \Phi(\xi, \eta, t) d \xi d \eta+\int_{0}^{t} F(t, \tau) \Phi(x, y, \tau) d \tau=f(x, y ; t) \\
& \quad=\pi \vartheta\left[\gamma(t)+\beta(t) x-f_{1}(x, y)\right] ;\left\{\Omega=\sqrt{x^{2}+y^{2}} \leq a ; \quad \vartheta=G(1-v)^{-1} ; 0 \leq h<1 ; h=\mu+0.5\right\}
\end{aligned}
$$


Under the dynamics conditions

$$
\iint_{\Omega} \Phi(\xi, \eta, t) d \xi d \eta=P(t), \quad \iint_{\Omega} \xi \eta \Phi(\xi, \eta, t) d \xi d \eta=M(t) ; \quad t \in[0, T] .
$$

The MIE (1), under the pressure and momentum conditions (2), is investigated from the axe- symmetric contact problem for an impressing stamp of angular form on a plane into a half space. The modules of elasticity of the plane is changing according to the power law $\sigma_{i}=\mathrm{K}_{o} \epsilon_{i}{ }^{h},(0 \leq h<1 ; h=\mu+0.5)$; where $\sigma_{i}, \epsilon_{i}$ are the stress and the strain rate intensities respectively, while $\mathrm{K}_{o}$ and $\mathrm{h}$ are physical constants. Assume that the stamp, has the equation formula $f_{1}(x, y)$ and is impressed into the lower surface by a variable force $\mathrm{P}(\mathrm{t})$, whose eccentricity of application $e(t)$, and a moment $\mathrm{M}(\mathrm{t})$ that cause rigid displacements $\gamma(\mathrm{t})$ and $\beta(\mathrm{t})$, where $\gamma(\mathrm{t})$ and $\beta(\mathrm{t}) \in \mathrm{C}[0, \mathrm{~T}]$. When the frictional forces in the domain of contact area $\Omega$, between the two surfaces, are so small that it can be neglected. In the interval time $t \in[0, T]$ the unknown function of the pressure between the two surfaces is variant, and such problem leads us to the integral equation.

In order to guarantee the existence of a unique solution of (1), we assume the following:

(i) $\|f(x, y, t)\|_{L(\Omega) \times C[0, T]}=H$, where $H$ is a constant, where the positive functions $\gamma(t)$ and $\beta(t)$ are continuous and belong to the class $C[0, T]$, while $f_{1}(x, y) \in L_{2}(\Omega)$.

(ii) The kernel of position satisfies in general, the discontinuity condition

$$
\left\{\iint_{\Omega} \mid L(\xi, \eta) \cdot k\left(x-\xi, y-\left.\eta\right|^{2} d \xi d \eta\right\}^{\frac{1}{2}} \leq M,(M \text { is a constant }) .\right.
$$

(iii) The positive function $F(t, \tau)$ with its partial derivatives, with respect to its variables, are continuous in the class $C[0, T]$; i.e. for all values of $t, \tau \in[0, T],|F(t, \tau)| \leq L_{1}<L$ and $\left|\frac{\partial F(t, \tau)}{\partial t}\right| \leq L_{2}<L ; L_{1}, L_{2}$, L are constants .

In the remainder part of this paper, we construct a series form solution in the space $L_{2}(\Omega) X C[0, T]$, for the MIE (1) in the treatment of various two-dimensional semi- symmetrical and three dimensions problems with mixed boundary conditions. The separation of variables is used to obtain system of VIEs of the second kind and FIE of the first kind. The mechanics problem and its physical properties is explained. The FIE of the first and second kind with logarithmic kernel is derived from the work. Moreover, the integro differential equation with Cauchy kernel is derived with its special cases. This equation has appeared in both combined infrared gaseous radiation and molecular condition, and elastic contact studies.

\section{Fundamental Theorems}

To separate the variables of Eq. (1) with the generalized kernel $L(\xi, \eta) K(x-\xi, y-\eta)$,we seek the general solution in the form, see (Abdou, M. A., 2002),

$$
\Phi(x, y ; t)=\Phi_{0}(x, y ; t)+\Phi_{1}(x, y ; t)
$$

Where, $\Phi_{0}(x, y, t)$ is the complementary solution and $\Phi_{1}(x, y, t)$ is the particular solution.

Using (3) in (1), we have

$$
\begin{gathered}
\iint_{\Omega} L(\xi, \eta)\left[(x-\xi)^{2}+(y-\eta)^{2}\right]^{-h} \Phi_{j}(\xi, \eta, t) d \xi d \eta+\int_{0}^{t} F(t, \tau) \Phi_{j}(x, y, \tau) d \tau \\
=\pi \vartheta \delta_{j}[\gamma(t)+\beta(t) x-f(x, y)], \quad j=0,1 ; \quad 0 \leq t \leq T<1,
\end{gathered}
$$

And for initial time, we have

$$
\iint_{\Omega} L(\xi, \eta)\left[(x-\xi)^{2}+(y-\eta)^{2}\right]^{-h} \Phi_{j}(\xi, \eta, 0) d \xi d \eta=\pi \vartheta \delta_{j}[\gamma(0)+\beta(0) x-f(x, y)] .
$$

Hence, from 4 and 5 , we obtain

$$
\begin{gathered}
\int_{\Omega} L(\xi, \eta)\left[(x-\xi)^{2}+(y-\eta)^{2}\right]^{-h}\left[\Phi_{j}(\xi, \eta, t)-\Phi_{j}(\xi, \eta, 0)\right] d \xi d \eta+\int_{0}^{t} F(t, \tau) \Phi_{j}(x, y, \tau) d \tau \\
=\pi \vartheta \delta_{j}[\gamma(t)+\beta(t) x-f(x, y)], \quad \delta_{j}= \begin{cases}1 & j=0 \\
0 & j=1\end{cases}
\end{gathered}
$$

Theorem 1(without proof): If the kernel of position $L(\xi, \eta) k(x-\xi, y-\eta)$ satisfies the discontinuity, condition (ii), then the integral operator

$$
K \Phi=\iint_{\Omega} L(\xi, \eta)\left[(x-\xi)^{2}+(y-\eta)^{2}\right]^{-h} \Phi(\xi, \eta, t) d \xi d \eta
$$

is compact in the Banach space $L_{2}(\Omega) \times C[0, T]$. Moreover, it is self-adjoint. 
Theorem (2) (without proof): For a symmetric and positive kernel,the integral operator (8)through the time interval $0 \leq t \leq T<1$ is compact and self-adjoint operator. Therefore, we can write it in the linear form

$$
\iint_{\Omega} L(\xi, \eta)\left[(x-\xi)^{2}+(y-\eta)^{2}\right]^{-h} \Phi(\xi, \eta, t) d \xi d \eta=\alpha_{k}(t) \Phi_{k}(x, y)
$$

where $\alpha_{n}$ and $\Phi_{n}$ are the eigenvalues and the eigenfunctions of the integral operator respectively.?

\section{General Solution of Mixed Integral Equation}

In this section, our aim is obtaining the general solution of Eq. (4) that has mixed formula of Fredholm integral term and Volterra integral term. Therefore, and in view of theorem (2), the solution of Eq. (4) can be expanded in a closed form of Fourier series in $L_{2}(\Omega) \times C[0, T]$ as a system of linear combination of eigenvalues and eigenfunctions:

$$
\Phi_{j}(x, y ; t)=\sum_{k=1}^{\infty}\left[A_{2 k}^{(j)}(t) \Phi_{2 k}(x, y)+A_{2 k-1}^{(j)}(t) \Phi_{2 k-1}(x, y)\right],
$$

where $\Phi_{2 k}(x, y), \Phi_{2 k-1}(x, y)$ are the even and odd functions respectively.

Using the formulas (9) and (10) in (6), the following results can be obtained

$$
\begin{gathered}
A_{k}^{(1)}(t)+\alpha_{k} \int_{0}^{t} A_{k}^{(1)}(\tau) F(t, \tau) d \tau=A_{k}^{(1)}(0) \quad(k \geq 1) \\
A_{2 k}^{(0)}(t)+\alpha_{2 k} \int_{0}^{t} A_{2 k}^{(0)}(\tau) F(t, \tau) d \tau=\pi \vartheta a_{2 k} b_{2 k}[\gamma(t)-\gamma(0)] \\
A_{2 k-1}^{(0)}(t)+\alpha_{2 k-1} \int_{0}^{t} A_{2 k-1}^{(0)}(\tau) F(t, \tau) d \tau=\pi \vartheta a_{2 k-1} b_{2 k-1}[\beta(t)-\beta(0)]
\end{gathered}
$$

where $\sum_{k=1}^{\infty} b_{2 k} \Phi_{2 k}=1, \quad \sum_{k=1}^{\infty} b_{2 k-1} \Phi_{2 k-1}=x, \quad\left(k \geq 1,0 \leq t \leq T<1, A_{2 k}^{(1)}(0)=A_{2 k-1}^{(1)}(0)=0\right)$

Equations (11) represent system of VIEs of the second kind with continuous kernel $F(t, \tau)$,the difference only in the free term. Therefore, every equation has a unique solution in the class $(C[0, T])$ depends on the free term $A_{k}^{(1)}(0), \gamma(\mathrm{t})$ and $\beta(\mathrm{t})$.

The constant value of $A_{k}^{(1)}(0)$ can be obtained directly from Eqs. (1), (4) in the form

$$
A_{k}^{(0)}(0)=\pi \vartheta \gamma(0) \alpha_{k}
$$

In view of Eqs.(8),(9) the general solution of Eq. (1) can be adapted in the form

$$
\Phi(x, y ; t)=\sum_{k=1}^{\infty}\left[A_{k}^{(0)}(t)+A_{k}^{(1)}(t)\right] \Phi_{k}(x, y)
$$

Where $A_{k}^{(0)}(t)$ and $A_{k}^{(1)}(t)$ must satisfy the inequality

$$
\sum_{k=1}^{\infty}\left[A_{k}^{(0)}(t)+A_{k}^{(1)}(t)\right]^{2}<\epsilon, \quad(\in<<1)
$$

and $\Phi_{k}(x, y)$ represents the eigenfunctions of the integral operator (9).

Theorem (3): If the inequality (13) is uniformly convergent and (12) holds. The series

(12) is uniformly convergent and Eq. (1) has a unique solution in $L_{2}(\Omega) X C[0, T]$.

Proof: Comparing the result of Eq. (12) with the first formula of (9) we get

$$
\left|A_{i}^{(0)}(t)\right| \leq \pi \vartheta\left|\alpha_{i}\right| \gamma(0) e^{\left(\left|\alpha_{i}\right| F\right) T}, \quad|F(t)|<F, \quad 0<\vartheta<1, \quad \gamma(0)<<1 .
$$

In addition,we have

$$
\left|A_{i}^{(1)}(t)\right| \leq \pi \vartheta\left|\alpha_{i}\right| \bar{\delta}^{-i} e^{\left(\left|\lambda_{i}\right| F\right) T}, \quad \overline{-}_{i}= \begin{cases}|\gamma(t)|=\gamma & \text { for odd values } \\ |\beta(t)|=\beta & \text { for even values }\end{cases}
$$


Finally, we get

$$
\left\{\sum_{i=1}^{\infty}\left[A_{i}^{(0)}(t)+A_{i}^{(1)}(t)\right]^{2}\right\}^{\frac{1}{2}} \leq \pi \vartheta B\left\{\sum_{i=1}^{\infty}\left(\left|\alpha_{i}\right| e^{\left|\lambda_{i}\right| F T}\right)^{2}\right\}^{\frac{1}{2}}
$$

The formula (14) represents the convergence condition.

The general solution of the second formula of VIEs (11) can be obtained in the form:

$$
A_{k}(t)=\exp \left(\alpha_{k} \int_{0}^{t} F(u, u) d u\right) \int_{0}^{t} \exp \left(-\alpha_{k} \int_{0}^{u} F(v, v) d v\right)\left(\pi \vartheta a_{k} b_{k} \frac{d w(u)}{d u}-\alpha_{k} \int_{0}^{u} A_{k}(\tau) \frac{\partial F(u, \tau)}{\partial u} d \tau\right) d u ; k=1,2, \ldots
$$

In (15), for even values of $k$ we take $w(t)=\gamma(t)$, and for odd values we take $w(t)=\beta(t)$.

\section{General Solution of Mixed Integral Equation:}

From the above discussion, the general solution of (1), can be adapted in the eigenvalues and eigenfunctions in the form:

$$
\Phi(x, y ; t)=\sum_{k=1}^{\infty} \alpha_{k} A_{k}(t) \Phi_{k}(x, y) .
$$

Where for even and oddnumbers we use, respectively the following conditions:

$$
\begin{gathered}
P(t)=P_{o}+\sum_{k=2,4} P_{k} \gamma_{k} \alpha_{k} A_{k}(t), \quad\left(P_{o} \text { is Cons. }\right), \\
M(t)=M_{o}+\sum_{k=1,3} M_{k} \beta_{k} \alpha_{k} A_{k}(t), \quad\left(M_{o} \text { is Cons. }\right) .
\end{gathered}
$$

The formula (16) represents the solution of the MIE of Eq. (1) in the form of eigenvalues $\alpha_{k} A_{k}(t)$, where $A_{k}(t)$ is the solution of Eq. (15), and eigenfunctions $\Phi_{k}(x, y)$. The value of the eigenfunctions are obtain when the kernel of (1) takes the generalized potential form. The boundary condition (17) is derived from (2) after using (16) in (2).

\section{Eigenvalues and Eigenfunctions of the Generalized Potential Function in Polar Coordinates}

To obtain the spectral relationships of Eq.(5), when $\delta=1$. Firstly, weuse the polar coordinates, $(x, \xi)=(r, \rho) \cos (\theta, \psi),(y, \eta)=$ $(r, \rho) \sin (\theta, \psi)$. Then,we use the separation variables method, $\Phi(x, y)=\Phi(r, \theta)=\Phi_{n}(r) \cos n \theta$.

Secondly, we consider the following famous two relations

$$
F_{1}\left(\alpha, \alpha+\frac{1}{2}-\beta ; \beta+\frac{1}{2} ; z^{2}\right)=(1+z)^{-2 \alpha} F_{1}\left(\alpha, \beta ; 2 \beta ; \frac{4 z}{(1+z)^{2}}\right),(|z|<1, \operatorname{Re} \alpha>0)
$$

Where $\Gamma(x)$ is the gamma function, $(\alpha)_{m} \frac{\Gamma(m+\alpha)}{\Gamma(\alpha)}$ is called Pachmmer symbol and $F_{1}(a, b, c ; z)$ is the Gauss hypergeometric function, see (Covalence, E. K., 1989).

$$
\int_{0}^{\infty} J_{\mu}(b x) J_{v}(a x) d x=a^{v} b^{-v-1} \frac{\Gamma\left(\frac{\mu+v+1}{2}\right)}{\Gamma(1+v) \Gamma\left(\frac{\mu-v+1}{2}\right)} \cdot F\left(\frac{\mu+v+1}{2}, \frac{v-\mu+1}{2}, v+1, a^{2} / b^{2}\right) .
$$

Which represents the integral relation between Bessel function $J_{m}(a x)$ of order $\mathrm{m}$ and hypergeometric function $F(\alpha, \beta ; \gamma ; z)$, see( Eq. 6.5121 pp. 680 of (Gradstein, I. M. \& Ryzhik, I. M, 1994)).

Hence, using the above two famous relations and following the same technique and way of (Abdou, M. A., 2002; Abdou, M. A., 2000), on noting the difference in notation,we obtain the following integral formula

$$
\int_{0}^{a} K_{n}^{h}(r, \rho) \phi(\rho) d \rho=\pi \vartheta f(r), \quad K_{n}^{h}(r, \rho)=\sqrt{r \rho} \int_{0}^{\infty} L(u) u^{2 h-1} J_{n}(\rho u) J_{n}(r u) d u .
$$

In the contact problems, in the theory of elasticity the function $L(u)$ must satisfy, see (Aleksandrov, V. M. \& Covalence, E. V., 1986).

$$
\begin{gathered}
L(u)=A u+O\left(u^{3}\right) ; \quad(u \rightarrow 0, A=C \text { onst. }>0), \\
L(u)=1+\sum_{i=1}^{\ell-1} \frac{B_{i}}{u^{i}}+O\left(\frac{1}{u^{\ell}}\right) \quad\left(u \rightarrow \infty, B_{i}=\text { constant }\right)
\end{gathered}
$$


The first formula of (18) represents FIE of the first kind with generalized potential kernel in the form of Weber-Sonien integral formula. It is not difficult to prove that the Weber-Sonien integral formula represents a non-homogeneous wave equation:

$$
\left(\frac{\partial^{2}}{\partial r^{2}}-\frac{\partial^{2}}{\partial \rho^{2}}\right) K_{n}^{h}(r, \rho)=\left[h_{n}(r)-h_{n}(\rho)\right] K_{n}^{h}(r, \rho) ; \quad h_{n}(v)=\left(n^{2}-\frac{1}{4}\right) v^{-2}
$$

Now, replacing the known function $f(r)$ in (18) by Jacobi polynomials i.e., letting $f(r)=P_{k}^{\left(n, \mu-\frac{1}{2}\right)}\left(1-2 r^{2}\right) ; \quad h=\mu+\frac{1}{2}, 0 \leq$ $\mu<1 / 2$; then using Krein's method, see (Ur Rehman, M. \& Khan, R. A., 2012) and after some integrations and algebraic relations, we arrive to the following formula

$$
\int_{0}^{1} \frac{K_{n}(u, v) \rho^{k+1}}{\left(1-v^{2}\right)^{\frac{1}{2}-\mu}} P_{k}^{\left(n, \mu-\frac{1}{2}\right)}\left(1-2 v^{2}\right) d v=\alpha_{k}^{n} u^{k} P_{k}^{\left(n, \mu-\frac{1}{2}\right)}\left(1-2 u^{2}\right), \quad(u=a r, v=a \rho)
$$

Where the eigenvalues $\alpha_{k}^{n}$ are given by

$$
\alpha_{k}^{n}=\pi \vartheta \frac{\Gamma\left(\mu+k+n+\frac{1}{2}\right) \Gamma\left(k+\mu+\frac{1}{2}\right) \Gamma\left(\frac{1}{2}-\mu\right)}{n !(k+n) ! \Gamma\left(\frac{1}{2}+\mu\right)} ; \quad(n=0,1,2,3, \ldots) .
$$

The formula (21) represents the spectral relations (solution) of (18) in the eigenvalues and eigenfunctions form.

Finally, the solution of (1) when the kernel of position takes the generalized potential form and the free term in the form of Jacobi polynomials, $P_{k}^{\left(k,-w^{-}\right)}\left(1-2 r^{2}\right)$ multiplying by a function of time $A_{k}(t)$ of (15), takes the form

$$
\Phi(u ; t)=\sum_{k=1}^{\infty} \alpha_{k}^{n} A_{k}(t) u^{k} P_{k}^{\left(n, \mu-\frac{1}{2}\right)}\left(1-2 u^{2}\right)
$$

where $A_{k}(t)$, is the solution of VIEs (11) and $\alpha_{k}$ is given by (22).

The formula (23) represents the general solution of (1) of semi-symmetric Hertz contact problem of frictionless impression of a rigid surface $(G, v)$ having an elastic material occupying the domain $\Omega=\left\{(x, y, z) \in \Omega: \sqrt{x^{2}+y^{2}} \leq a, z=0\right\}$ in three dimensions in prance of time and under the dynamic conditions of pressure and momentum.

\section{Applications of Spectral Relations and Special Cases:}

In this section, many applications of spectral relations, in mathematical physics problems and special cases can be derived from (23).

\section{Case (i): Semi - symmetric contact problem, in three dimensional with potential kernel, in presence of time:}

When $L(x, y)=1, h=0.5$, theMIE(1) takes the form

$$
\begin{gathered}
\iint_{\Omega} \frac{\Phi(\xi, \eta, t)}{R} d \xi d \eta+\int_{0}^{t} F(t, \tau) \Phi(x, y, \tau) d \tau=\pi \vartheta[\gamma(t)+\beta(t) x-f(x, y)] \\
; R=\sqrt{(x-\xi)^{2}+(y-\eta)^{2}} \quad \vartheta=G(1-v)^{-1} .
\end{gathered}
$$

The IE (24) constructs the solution of the action problem of an inclined ring stamp, $a \leq r \leq b$, on an elastic half-space that is free from external forces and the frictional forces in the domain of contact area between the stamp and half-space are neglected.

The potential kernel can be written in the form

$$
K_{n}(u, v)=\frac{\sqrt{\pi} \Gamma(n+1 / 2) e^{2 k}}{2^{2 n-1} n !(u+v)} F\left(n+\frac{1}{2}, n+\frac{1}{2}, 2 n+1, e^{2}\right), \quad\left(e=E\left(\frac{2 \sqrt{u v}}{u+v}\right)\right)
$$

Here, in (25), $\Gamma(\mathrm{x})$ is the Gamma function and $E\left(\frac{2 \sqrt{r \rho}}{r+\rho}\right)$ is called the complete elliptic integral form.

Using the integral representation of hypergeometric series 9.111, pp.1054 of (Gradstein, I. M. \& Ryzhik, I. M, 1994).

$$
F(\alpha, \delta, \gamma, z)=\frac{1}{\beta(\delta, \gamma-\delta)} \int_{0}^{t} t^{\delta-1}(1-t)^{\gamma-\delta-1}(1-t z)^{-\alpha} d t
$$

where $(\beta$ is Beta function $\operatorname{Re} \gamma>\operatorname{Re} \delta>0)$ 


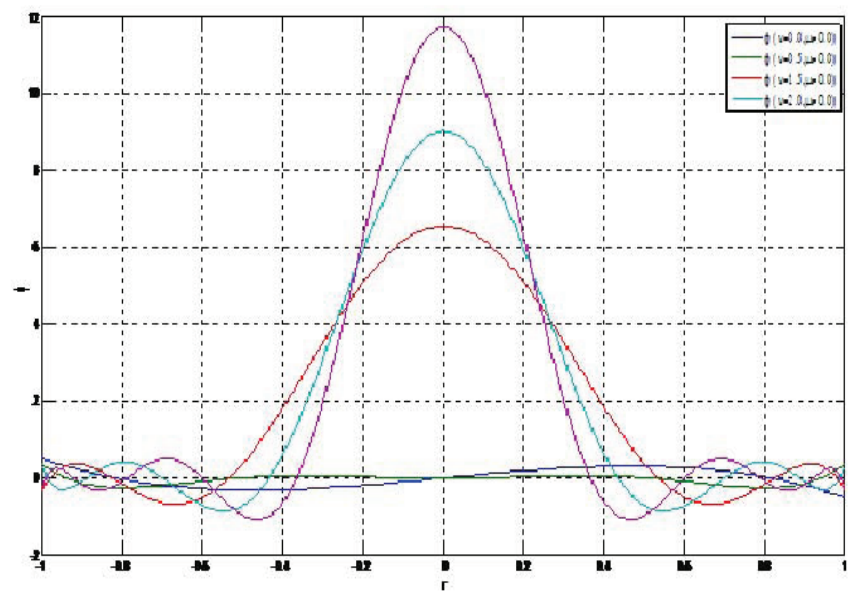

Figure 1. The solution of the MIE with potential kernel for some different values $k, u$

We can rewrite (26), for any higher order harmonic, $k \geq 0$, in the integral elliptic form

$$
K_{n}(u, v)=\frac{e^{2 n}}{\pi(u+v)} \int_{0}^{1} z^{n-\frac{1}{2}}(1-z)^{n-\frac{1}{2}}\left(1-e^{2} z\right)^{-n-\frac{1}{2}} d z=\sqrt{u v} \int_{0}^{\infty} J_{n}(u \tau) J_{n}(v \tau) d \tau .
$$

The spectral relations (21) take the form

$$
\int_{0}^{1} K_{n}(u, v) \frac{v^{n+1}}{\sqrt{1-v^{2}}} P_{k}^{\left(n,-\frac{1}{2}\right)}\left(1-2 v^{2}\right) d v=\left\{\begin{array}{c}
\pi \vartheta \frac{\Gamma\left(k+n+\frac{1}{2}\right) \Gamma\left(k+\frac{1}{2}\right)}{n !(k+n) !} u^{n} P_{k}^{\left(n,-\frac{1}{2}\right)}\left(1-2 u^{2}\right), u<1 \\
\pi \vartheta(-1)^{k} \frac{\Gamma\left(k+n+\frac{1}{2}\right) \Gamma\left(k+\frac{1}{2}\right)}{n !(k+n) !} u^{n} P_{k}^{\left(-\frac{1}{2}, n\right)}\left(2 u^{2}-1\right), u>1 .
\end{array},\right.
$$

Hence, the complete solution of the problem is:

$$
\Phi(u ; t)=\sum_{k=1}^{\infty} A_{k}(t)\left\{\begin{array}{cc}
\pi \vartheta \frac{\Gamma\left(k+n+\frac{1}{2}\right) \Gamma\left(k+\frac{1}{2}\right)}{n !(k+n) !} u^{n} P_{k}^{\left(n,-\frac{1}{2}\right)}\left(1-2 u^{2}\right) & , u<1 \\
\pi \vartheta(-1)^{k} \frac{\Gamma\left(k+n+\frac{1}{2}\right) \Gamma\left(k+\frac{1}{2}\right)}{n !(k+n) !} u^{n} P_{k}^{\left(-\frac{1}{2}, n\right)}\left(2 u^{2}-1\right) & , u>1
\end{array}\right.
$$

\section{Case (ii): Three-dimensional semi symmetric Hertz contact problem with elliptic kernel:}

Let, in (29) $n=0$ we have the solution of MIE (1), under the conditions (2) with elliptic kernel in position. The importance of the elliptic kernel came from the work of Covalence (Covalence, E. K., 1989), who developed the Fredholm equation of the first kind for the mechanics mixed problem of continuous media and obtained its approximate solution.

The general solution, in this case, takes the form

$$
\Phi(u ; t)=\sum_{k=1}^{\infty} A_{k}(t)\left\{\begin{array}{c}
\pi \vartheta \pi\left[\frac{\Gamma\left(k+\frac{1}{2}\right)}{k !}\right]^{2} P_{k}^{\left(0,-\frac{1}{2}\right)}\left(1-2 r^{2}\right), \quad r<1 \\
\pi \vartheta(-1)^{k} \pi\left[\frac{\Gamma\left(k+\frac{1}{2}\right)}{k !}\right]^{2} P_{k}^{\left(-\frac{1}{2}, 0\right)}\left(2 r^{2}-1\right), R>1
\end{array}\right.
$$




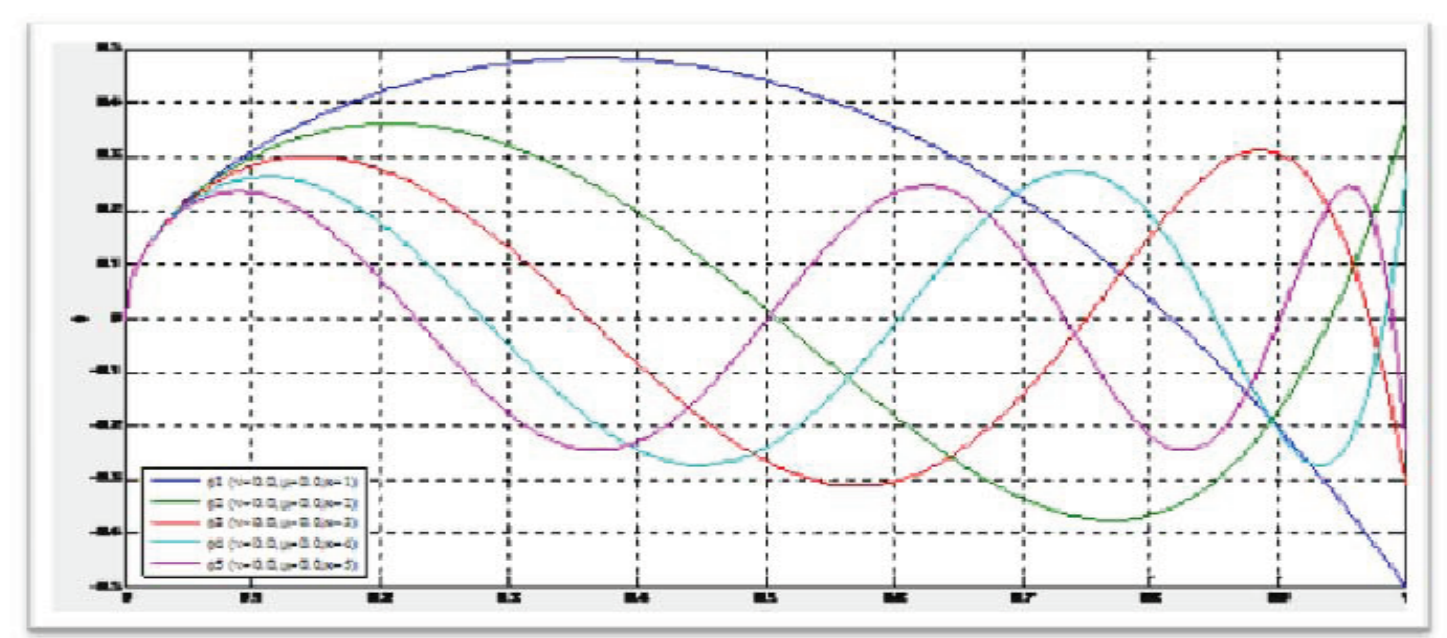

Figure 2. The solution of Eq.(30) for different values of $k$

\section{Case (iii): One dimension mixed integral equation with Carleman kernel:}

In (Arytunian, N. K. H., 1959), Arytunian has shown that the plane contact problem of the nonlinear theory of plasticity, in its first approximation, reduce to Fredholm integral equation of the first kind with Carleman kernel. For $n=\frac{1}{2}$, and with the aid of the following relation, see (Gradstein, I. M. \& Ryzhik, I. M, 1994)

$$
{ }_{2} F_{1}\left(-\frac{m-1}{2},-\frac{m-2}{2} ; \frac{3}{2} ; \frac{z^{2}}{t^{2}}\right)=\frac{(t+z)^{m}-(t-z)^{m}}{2 m z t^{m-1}}
$$

The general solution of the MIE with Carleman function for $n=1 / 2$ is given as

$$
\Phi(u ; t)=\sum_{k=1}^{\infty} A_{k}(t)\left\{\begin{array}{c}
\pi \vartheta \frac{\Gamma(\mu+k+1) \Gamma\left(k+\mu+\frac{1}{2}\right) \Gamma\left(\frac{1}{2}-\mu\right)}{k ! \Gamma\left(k+\frac{3}{2}\right) \Gamma\left(\frac{1}{2}+\mu\right)} \sqrt{r} P_{k}^{\left(\frac{1}{2}, \mu-\frac{1}{2}\right)}\left(1-2 r^{2}\right), r<1 \\
\pi \vartheta(-1)^{k} \frac{\Gamma(\mu+k+1) \Gamma\left(k+\mu+\frac{1}{2}\right) \Gamma\left(\frac{1}{2}-\mu\right)}{k ! \Gamma\left(k+\frac{3}{2}\right) \Gamma\left(\frac{1}{2}+\mu\right)} \sqrt{r} P_{k}^{\left(\mu-\frac{1}{2}, \frac{1}{2}\right)}\left(2 r^{2}-1\right), r>1
\end{array}\right.
$$

While, the spectral relations when $n=-\frac{1}{2}$ are

$$
\Phi(u ; t)=\sum_{k=1}^{\infty} A_{k}(t)\left\{\begin{array}{c}
\pi \vartheta \frac{\Gamma(\mu+k) \Gamma\left(k+\mu+\frac{1}{2}\right) \Gamma\left(\frac{1}{2}-\mu\right)}{k ! \Gamma\left(k+\frac{1}{2}\right) \Gamma\left(\frac{1}{2}+\mu\right)} r^{-\frac{1}{2}} P_{k}^{\left(-\frac{1}{2}, \mu-\frac{1}{2}\right)}\left(1-2 r^{2}\right), r<1 \\
\pi \vartheta(-1)^{k} \frac{\Gamma(\mu+k) \Gamma\left(k+\mu+\frac{1}{2}\right) \Gamma\left(\frac{1}{2}-\mu\right)}{k ! \Gamma\left(k+\frac{1}{2}\right) \Gamma\left(\frac{1}{2}+\mu\right)} r^{-\frac{1}{2}} P_{k}^{\left(\mu-\frac{1}{2},-\frac{1}{2}\right)}\left(2 r^{2}-1\right), r>1
\end{array}\right.
$$

Case (iv): One dimension mixed integral equation with logarithmic kernel:

When $\mu=0, m=\frac{1}{2}$,

$$
\Phi(u ; t)=\sum_{k=1}^{\infty} A_{k}(t)\left\{\begin{array}{cc}
\frac{2 \pi \vartheta}{2 n+1} \sqrt{r} P_{k}^{\left(\frac{1}{2},-\frac{1}{2}\right)}\left(1-2 r^{2}\right), & r<1 \\
\frac{2(-1)^{k} \vartheta \pi}{2 n+1} \sqrt{r} P_{k}^{\left(-\frac{1}{2}, \frac{1}{2}\right)}\left(2 r^{2}-1\right), & r>1
\end{array}\right.
$$




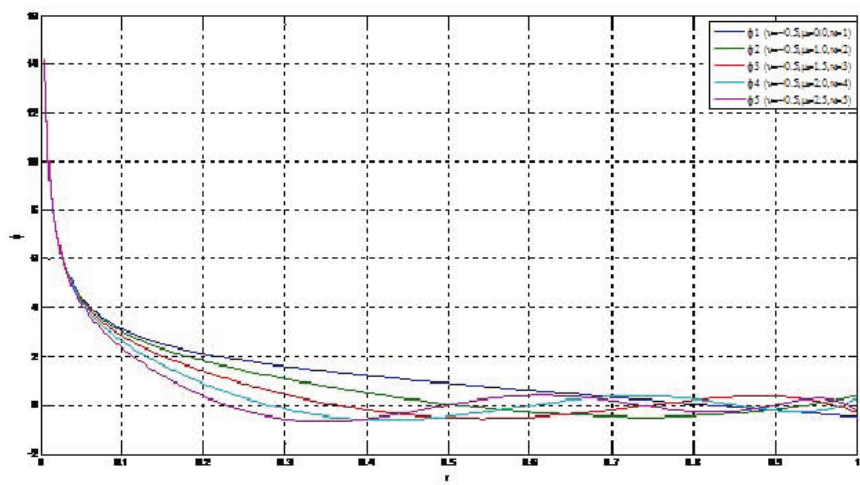

Figure 3. The solution of MIE with Carleman kernel for $n=1 / 2$ in Fig. 3 and $n=-1 / 2$ in Fig. 4

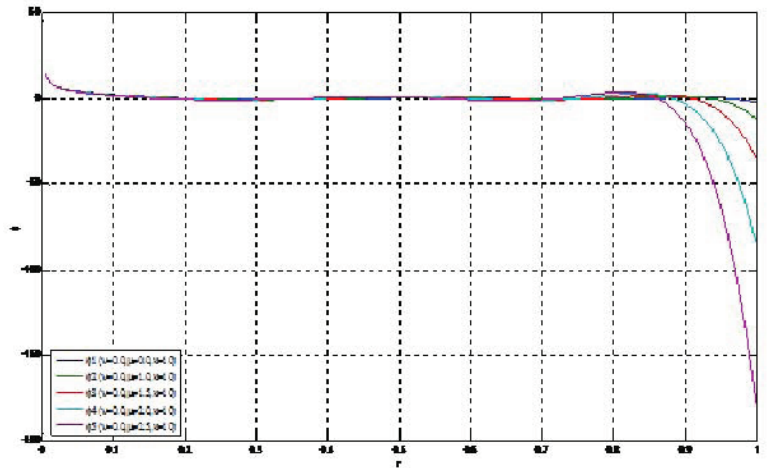

Figure 4. Solution for different values of $\mu$ 


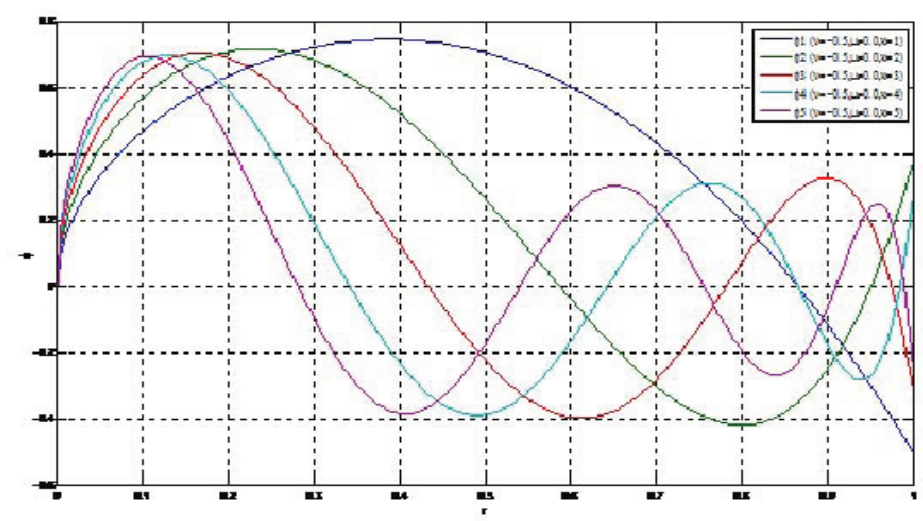

Figure 5. The solution of MIE (Eq.(33)) with logarithmic kernel for $n=1 / 2$
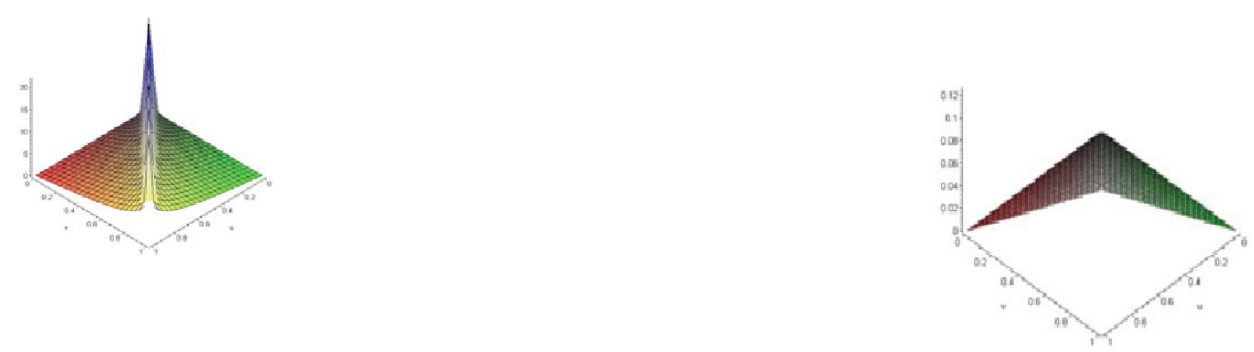

Figure 6. case $\mathrm{h}=0$

Figure 7. case $\mathrm{h}=0.1$
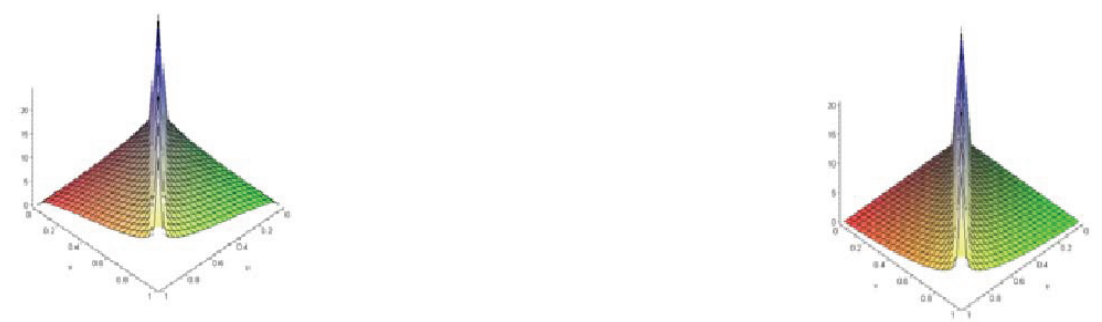

Figure 8 . case $h=0.5, n=0$

Figure 9. case $h=0.5, n=1$

Many special cases can be derived from the generalized kernel (18) as the following:

(i) When $h=0$, and $n= \pm 0.5$, we have the logarithmic kernel, see Fig.5, 
(ii) When $0 \leq h<1, n= \pm 0.5$, we have Carleman function, see Fig.3 and Fig. (4).

(iii) When $h=n=0$, we have the elliptic kernel, see Fig.2.

(iv) When $h=1 / 2$, we have the potential kernel, see Fig.1.

\section{Conclusions}

From the above results and discussions, the following may be concluded:

1-The MIE (1), under the conditions (2) represents the three dimensional semi-symmetric Hertz contact problemof frictionless impression of a rigid surface $(G, v)$ having an elastic material occupying the domain $\Omega=\left\{\sqrt{x^{2}+y^{2}} \leq a, z=0\right\}$ where $f_{1}(x, y) \in L_{2}(\Omega)$ describing the surface of stamp. This stamp is impressed into an elastic layer surface, plane, by a variable known force $P(t)$ whose eccentricity of application $e(t)$ and a variable known momentum $M(t), 0 \leq t \leq T<1$, that cause rigid displacements $\gamma(t)$ and $\beta(t) x$, respectively. Here, the force $F(t, \tau), t, \tau \in[0, T]$, represents the characterized resistance function of the material,$G$ is the displacement magnitude, $v$ is Poisson's coefficient, and $\Phi(x, y, t)$ represents the unknown normal stresses between the stamp and the surface.

2-The problems of elastic material of strip $\left(\mathrm{G}_{1}, v_{1}\right)$ of thickness $\mathrm{h}$, which occupies the domain $|x|<\infty$ and lies without frictions on a rigid elastic layer $\left(\mathrm{G}_{2}, v_{2}\right)$ of function $f(x)$. If a stamp of width $2 \mathrm{a}$ impressed into the strip $\mathrm{y}=\mathrm{h}$ by a variable force with respect to time $\mathrm{P}(\mathrm{t}), \mathrm{t} \in[0, T]$ and a moment $M(t)$, these kind of problems reduce to a MIE of the first kind and are considered as special cases of this work, see (Ur Rehman, M. \& Khan, R. A., 2012; Aleksandrov, V. M. \& Covalence, E. V., 1989).

3-The displacement problems of anti-plane deformation of an infinite rigid strip with width $2 a_{-}$, putting on an elastic layer of thickness $h$ is considered as a special case of this work when $t=1_{-}, F(t, \tau)=1_{-}, f(x, t)=\mathrm{H}_{-}$and $\phi(x, 1)=\psi(x){ }_{-}$. Here, $H_{-}$represents the displacement magnitude and $\psi(x)_{-}$the unknown function represents the displacement stress, see (Abdou, M. A., 2002).

4-The FIE of the first kind with a logarithmic kernel, Carleman kernel, complete elliptic kernel and potential kernel is included as special cases of this work.

5-The contact problem of the zero harmonic symmetric kernels is included as a special case when $\mathrm{n}=0$, see Eqs. (12), (13). Also the contact problem of the first and higher order harmonic, $n=1,2, \ldots$,is included as a special case.

6-The problems of infinite rigid strip with width $2 a$ impressed in a viscous liquid layer of thickness $h$, when the strip has a velocity resulting from the impulsive force $V=V_{0} e^{-i w t}, i=\sqrt{-1}$, where $V_{0}$ is the constant velocity, $w$ is the angular velocity resulting rotating the strip about z-axis are considered as special case of this work, when $F(t, \tau)={ }_{-}$constant and $t=1_{-}$, see (Ur Rehman, M. \& Khan, R. A., 2012).

7-In the discussion of (6), when $h \rightarrow \infty$, i.e. the depth of the liquid ( fluid mechanics ) or the thickness of elastic material (contact problem ) becomes an infinite, see (Aleksandrov, V. M. \& Covalence, E. V., 1989).

8-From this work, we can derive many spectral relations that are very important in mathematical physics problems.

i-The spectral relations for the integral equation (18) in the Legendre polynomials form take the forms

$$
\int_{0}^{1} K_{n}(r, \rho) P_{m}^{n}(\rho)\left(1-\rho^{2}\right)^{h-1} \rho^{n+1} d \rho=\left\{\begin{array}{l}
A_{n m}^{h} r^{n} P_{n}^{h}(r) \\
B_{n m}^{h} Q_{n}^{(h-1, n)}\left(2 r^{2}-1\right) \quad r>1
\end{array}\right.
$$

where

$$
\begin{gathered}
A_{n k}^{h}=2^{2(h-1)} \pi \vartheta \Gamma(k+n+h) \Gamma(k+h)[\Gamma(k+n+1) m !]^{-1},\left(P_{k}^{h}(r)=P_{k}^{(n, h-1)}\left(1-2 r^{2}\right)\right) \\
B_{n k}^{h}=(-1)^{k} 2^{2 h-1}(\sin \pi h) \Gamma(k+n+h) \Gamma(k+h)[\pi \Gamma(k+n+1) m !]^{-1}, k=1,2, \ldots
\end{gathered}
$$

Here $\Gamma($.$) is the Gamma function while P_{m}^{(\alpha, \beta)}(x), Q_{n}^{(\alpha, \beta)}(x)$ are the Jacobi polynomial of the first and second type respectively

ii- The spectral relations for the elliptic kernel are

$$
\int_{0}^{1} K_{0}(r, \rho) \frac{\rho}{\sqrt{1-\rho^{2}}} P_{k}^{\left(0,-\frac{1}{2}\right)}\left(1-2 \rho^{2}\right) d \rho=\left\{\begin{array}{c}
\pi \vartheta\left[\frac{\Gamma\left(k+\frac{1}{2}\right)}{k !}\right]^{2} P_{k}^{\left(0,-\frac{1}{2}\right)}\left(1-2 r^{2}\right), \quad r<1, \\
\pi \vartheta(-1)^{k}\left[\frac{\Gamma\left(k+\frac{1}{2}\right)}{k !}\right]^{2} P_{k}^{\left(-\frac{1}{2}, 0\right)}\left(2 r^{2}-1\right), \quad r>1,
\end{array}\right.
$$


iii- The spectral relations when $n=\frac{1}{2}$ are

$$
\begin{gathered}
\int_{0}^{1} K_{\frac{1}{2}}(u, v) v^{\frac{3}{2}}\left(1-v^{2}\right)^{\mu-\frac{1}{2}} P_{k}^{\left(\frac{1}{2}, \mu-\frac{1}{2}\right)}\left(1-2 v^{2}\right) d v=\left\{\begin{array}{cc}
D_{k} \sqrt{u} P_{k}^{\left(\frac{1}{2}, \mu-\frac{1}{2}\right)}\left(1-2 u^{2}\right), & u<1 \\
(-1)^{k} D_{k} \sqrt{u} P_{k}^{\left(\mu-\frac{1}{2}, \frac{1}{2}\right)}\left(2 u^{2}-1\right), \quad u>1
\end{array}\right. \\
D_{k}=\pi \vartheta \frac{\Gamma(\mu+k+1) \Gamma\left(k+\mu+\frac{1}{2}\right) \Gamma\left(\frac{1}{2}-\mu\right)}{k ! \Gamma\left(k+\frac{3}{2}\right) \Gamma\left(\frac{1}{2}+\mu\right)} .
\end{gathered}
$$

iv- The spectral relations when $n=-\frac{1}{2}$ are

$$
\begin{gathered}
\int_{0}^{1} K_{-\frac{1}{2}}(u, v) v^{\frac{1}{2}}\left(1-v^{2}\right)^{\mu-\frac{1}{2}} P_{k}^{\left(-\frac{1}{2}, \mu-\frac{1}{2}\right)}\left(1-2 v^{2}\right) d v=\left\{\begin{array}{c}
D_{k}^{*} u^{-\frac{1}{2}} P_{k}^{\left(-\frac{1}{2}, \mu-\frac{1}{2}\right)}\left(1-2 u^{2}\right), \quad u<1 \\
(-1)^{k} D_{k}^{*} u^{-\frac{1}{2}} P_{k}^{\left(\mu-\frac{1}{2},-\frac{1}{2}\right)}\left(2 u^{2}-1\right), \quad u>1
\end{array}\right. \\
D_{k}^{*}=\pi \vartheta \frac{\Gamma(\mu+k) \Gamma\left(k+\mu+\frac{1}{2}\right) \Gamma\left(\frac{1}{2}-\mu\right)}{k ! \Gamma\left(k+\frac{1}{2}\right) \Gamma\left(\frac{1}{2}+\mu\right)} .
\end{gathered}
$$

iiv-The spectral relations when $\mu=0, n=\frac{1}{2}$ are

$$
\int_{0}^{1} K_{\frac{1}{2}}(r, \rho) \frac{\rho^{\frac{3}{2}}}{\sqrt{1-\rho^{2}}} P_{n}^{\left(\frac{1}{2},-\frac{1}{2}\right)}\left(1-2 \rho^{2}\right) d \rho= \begin{cases}A_{n} \sqrt{r} P_{n}^{\left(\frac{1}{2},-\frac{1}{2}\right)}\left(1-2 r^{2}\right), & r<1 \\ B_{n} \sqrt{r} P_{n}^{\left(-\frac{1}{2}, \frac{1}{2}\right)}\left(2 r^{2}-1\right), & r>1\end{cases}
$$

\section{References}

Abdou, M. A. (2000). Fredholm integral equation with potential kernel and its structure resolvent. Appl. Math.Compute., 107, 169-180. https://doi.org/10.1016/S0096-3003(98)10132-7

Abdou, M. A. (2002). Fredholm - Volterra equation of the first kind and contact problem. Appl. Math. Compute. 125, 177 -193. https://doi.org/10.1016/S0096-3003(00)00118-1

Abdou, M. A. (2002). Fredholm?Volterra integral equation and generalized potential kernel. J. Appl. Math.Compute., 131, 81-94. https://doi.org/10.1016/S0096-3003(01)00128-X

Abdou, M. A. (2002). Integral equation of mixed type and integrals of orthogonal polynomials. J. Comp. Appl. Math., 138, 273 - 285. https://doi.org/10.1016/S0377-0427(01)00377-6

Aleksandrov, V. M., \& Covalence, E. V. (1986). Problems in Mechanics Media with Mixed Boundary Conditions, Nuka Moscow

Aleksandrov, V. M., \& Covalence, E. V. (1989). Mathematical method in the displacement problem. Inzh, Zh, Mekh. Tverd.Tela., 2, 77-89.

Arytunian, N. K. H. (1959). A plane contact problem of creep theory. Appl. Math. Mech. 23, (2)13-19.

Atkinson, K. E. (1976). A Survey of Numerical Method for the Solution of Fredholm Integral Equation of the Second Kind. SIAM, Philadelphia.

Covalence, E. K. (1989). Some approximate methods solving integral equations of mixed problems. Appl. Math. Mech., $53,85-92$.

Delves, L. M., \& Mohamed, J. L. (1985). Computational Methods for Integral Equations. Philadelphia.

Diego, T., \& Lima, P. (2008). Super convergence of collocation methods for class of weakly singular integral equations. J. Cam. Appl. Math., 218, 307-316.

Dzhuraev. (1992). Methods of Singular Integral Equations. London, New York.

Geng, F. Z., \& Qian, S. P. (2015). Modi_ed reproducing kernel method for singularly perturbed boundary value problems with a delay. Applied Mathematical Modeling, 39, 5592-5597. 
Golberg, M. A. (1990). Numerical Solution of Integral Equation. Plenum, N. Y. https://doi.org/10.1007/978-1-48992593-0

Gouyandeh, Z., Allahviranloo, T., \& Armand, A. (2016). Numerical solution of nonlinear Volterra-Fredholm-Hammerstein integral equations via Tau-collocation method with convergence analysis, Journal of Computational and Applied Mathematics, 308, 435-446.

Gradstein, I. M., \& Ryzhik, I. M. (1994). Tables of Integrals, Series and Products Academic Press, Inc.: New York.

Hochstadt, H. (1971). Integral equations. N.Y. London

Linz, P. (1985). Analytical an numerical methods for Volterra equations, SIAM, Studies in Applied Mathematics.

Mohammadi, M., \& Mokhtari, R. (2011). Solving the generalized regularized long wave equation on the basis of a reproducing kernel space. Journal of Computational and Applied Mathematics, 235, 4003-4014.

Mohammadi, M., \& Mokhtari, R. (2013). A new algorithm for solving nonlinear Shrodinger equation in the reproducing kernel space. Iranian Journal of Science E Technology, 37, 513-526.

Tricomi, F. G. (1985). Integral equations. N. Y.

Ordokhani, Y., \& Dehestani, H. (2010). An application of walsh functions for Fredholm- Ham- merstein integrodifferential equations. Int. J. Contemp. Math. Sciences, 5, 1055-1063.

Ordokhani, Y. (2013). An Application of Bessel function for Solving Nonlinear Fredholm- Volterra-Hammerstein Integrodifferential Equations, J. Sci. Kharazmi University, 13, 347-362.

Parand, K., \& Rad, J. A. (2012). Numerical solution of nonlinear Volterra-Fredholm- Hammerstein integral equations via collocation method based on radial basis functions. Applied Mathematics and Computation, 218, 5292-5309. https://doi.org/10.1016/j.amc.2011.11.013

Popov, G. Ya. (1982). Contact problems for a linearly deformable base, Kiev, Odessa.

Saeedi, L., Tari, A., \& Masuleh, S. H. (2013). Numerical solution of a class of the nonlinear Volterra integro-differential equations. J. Appl. Math. Informatics, 31, 65-77. https://doi.org/10.14317/jami.2013.065

Schiavone, P., Constanda, C., \& Mioduchowski, A. (2002). Integral Methods in Science and Engineering. Birkhauser Boston.

Turkyilmazoglu, M. (2014). High-order nonlinear Volterra-Fredholm-Hammerstein integro-differential equations and their effective computation, Applied Mathematics and Computation, 247, 410-416.

Ur Rehman, M., \& Khan, R. A. (2012). A numerical method for solving boundary value problems for fractional differential equations. Appl. Math. Model., 36, 894-907. https://doi.org/10.1016/j.apm.2011.07.045

\section{Copyrights}

Copyright for this article is retained by the author(s), with first publication rights granted to the journal.

This is an open-access article distributed under the terms and conditions of the Creative Commons Attribution license (http://creativecommons.org/licenses/by/4.0/). 